UG-10/95

\title{
$(2+1)$-Dimensional QED with Dynamically Massive Fermions in the Vacuum Polarization
}

\author{
V.P. Gusynin ${ }^{1,2}$, A.H. Hams ${ }^{1}$ and M. Reenders ${ }^{1}$ \\ ${ }^{1}$ Institute for Theoretical Physics, \\ University of Groningen, 9747 AG Groningen, The Netherlands \\ 2 Bogolyubov Institute for Theoretical Physics, \\ National Academy of Sciences of Ukraine, 252143 Kiev, Ukraine
}

(September 19, 1995)

\begin{abstract}
We study chiral symmetry breaking in $\mathrm{QED}_{3}$ with $N_{f}$ flavors of fourcomponent fermions. A closed system of Schwinger-Dyson equations for fermion and photon propagators and the full fermion-photon vertex is proposed, which is consistent with the Ward-Takahashi identity. A simplified version of that set of equations is reduced (in nonlocal gauge) to the equation for a dynamical fermion mass function, where the one-loop vacuum polarization with dynamically massive fermions has been taken into account. The linearized equation for the fermion mass function is analyzed in real space. The analytical solution is compared with the results of numerical calculations of the nonlinear integral equation in momentum space.
\end{abstract}

11.30.Qc, 11.30.Rd, 11.10.Kk, 11.15.Tk

Typeset using REVTEX 


\section{INTRODUCTION}

Quantum electrodynamics in one temporal, and two spatial dimensions with $N_{f}$ flavors of four-component Dirac fermions $\left(\mathrm{QED}_{3}\right)$ continues to attract attention as a useful field-theoretical model for studying such phenomena as confinement and chiral symmetry breaking $(\chi \mathrm{SB})$ which are out of the realm of perturbation theory. The model has properties reminiscent of QCD and other four-dimensional gauge theories. Thus it has an intrinsic dimensionful parameter $e^{2}$, the coupling constant, that plays a role similar to the QCD scale $\Lambda$, and the effective coupling $\alpha(q)$ approaches zero at large momenta $q$ [1]. Studying SchwingerDyson equation (SDE) for the fermion self-energy under the bare vertex approximation in the Landau gauge, Appelquist, Nash and Wijewardhana [2] have shown the existence of a finite critical number of fermions, $N_{c}$, below which the chiral symmetry is broken and fermions acquire a dynamical mass. In the vicinity of $N_{c}$ this mass is exponentially small with respect to the natural dimensionful parameter $e^{2}$, showing how a hierarchy of scales can occur in gauge theories. These results were confirmed by Dagotto, Kogut, Kocić [3,4] in their Monte-Carlo simulations of non-compact lattice $\mathrm{QED}_{3}$.

Continuum studies of $\chi S B$ use SDE which, being an infinite chain of equations, must be truncated in some way. The bare vertex approximation was criticised $[5,6]$ as not being consistent with the Ward-Takahashi (WT) identity. An effective way to truncate SDE is to make an ansatz for the vertex satisfying certain criteria [7] (for a review of SDE in $\mathrm{QED}_{3}$ see [8]). While at the present time the latter approach seems to be the most effective way to satisfy WT identity under truncation, it does not shed much light on an approximation from the physical point of view. Ideally, one should solve SDE for the vertex itself, but that is quite a formidable task. However, in the second section we propose one possible truncation scheme consistent with WT identity not mentioned before in the literature.

Another important point in studying the $N_{f}$ dependence of $\chi \mathrm{SB}$ is that it is necessary to insert vacuum polarization effects into the equation for the fermion dynamical mass (in quenched approximation, chiral symmetry is broken for any $\left.N_{f}[9,10,4]\right)$. In $1 / N_{f}$ expansion the one-loop vacuum polarization with massless fermions is used. However, as was mentioned in $[11,12]$, the inclusion of massive fermions changes drastically the infrared behavior of the model, leading to the appearance of a confining (logarithmic) term in the potential at large distances. To take into account this confining nature of the model one can study the coupled system of SDE for the fermion self-energy, the photon polarization tensor and the vertex (for example, to study the coupled system of equations for them mentioned above). In this paper we chose another approach, replacing the fermion dynamical mass function in the polarization tensor by a constant dynamical mass of a fermion. While keeping the confinement property of the model this makes analysis much simpler. Because this approximation just corresponds to using the bare vertex, in order to overcome the inconsistency with the WT identity (or, at least, minimize its violation) we selected a nonlocal gauge $[13,14]$ to keep the fermion wave function $Z(p) \equiv 1$.

In the third section we reconsider the analysis of linearized SDE for a fermion mass function, converting it into a Schrödinger-like equation in real Euclidean space with an effective potential. For the vacuum polarization with massless fermions this potential behaves as $-\lambda / r^{2}$ at large $r\left(\lambda=32 / 3 \pi^{2} N_{f}\right)$. From the theory of the Schrödinger equation for such kinds of potentials, it is known that it has an infinite number of bound states if 
$\lambda>1 / 4\left(N_{f}<N_{c}=128 / 3 \pi^{2}\right)$. However a finite number of bound states might exist for $\lambda<1 / 4$ as well. We prove that for the potential under consideration there are no bound states for $\lambda<1 / 4$, which means that the critical number $N_{c}$ does indeed correspond to the appearance of an infinite number of bound states.

In the case of massive fermions, the vacuum polarization leads to a Coulomb tail in an effective potential at large $r$, however, as we show in Section 4, this does not alter the exponential-type behavior of a dynamical mass near the critical $N_{c}$. This is, of course, in agreement with bifurcation analysis (see, for example, $[15,16]$ ). The main difference between a vacuum polarization with massive or with massless fermions lies in an overall scaling factor, which is larger for massive fermion loops in vacuum polarization. From the physical point of view it means that chiral symmetry breaking takes place essentially at intermediate distances $1 / \alpha<r<1 / m\left(\alpha=e^{2} N_{f} / 8\right)$ and is not influenced much by large distances, where the confinement property of the model exhibits itself. This situation is similar to that in $\mathrm{QCD}_{4}[17,18]$, where the chiral symmetry breaking scale is also different from the scale of confinement.

The real space approach allows us to get a good analytical solution, fitting nicely computer calculations when $N_{f}$ is close to $N_{c}$. In Section 5 we return to momentum space to analyze nonlinear SDE for a fermion dynamical mass function. We give the results of computer calculations of the dynamical mass function, which show very convincingly how a new energy scale (mass of a fermion) appears that is much smaller than the natural scale $\left(e^{2}\right)$ of the model. This might be important for a better understanding of a hierarchy of scales in unified theories.

\section{TRUNCATION OF SCHWINGER-DYSON EQUATIONS}

In $\mathrm{QED}_{3}$ the Schwinger-Dyson equation for the fermion propagator in Minkowski space is given by

$$
S^{-1}(p)=\hat{p}-\frac{i e^{2}}{(2 \pi)^{3}} \int_{M} \mathrm{~d}^{3} k \gamma^{\mu} S(k+p) \Gamma^{\nu}(k+p, p) D_{\mu \nu}(k),
$$

and the SDE for the photon propagator is

$$
D_{\mu \nu}^{-1}(q)=D_{b_{\mu \nu}}^{-1}(q)+\Pi_{\mu \nu}(q),
$$

where

$$
\Pi^{\mu \nu}(q)=\frac{i e^{2}}{(2 \pi)^{3}} \int_{M} \mathrm{~d}^{3} k \operatorname{Tr}\left[\gamma^{\mu} S(k) \Gamma^{\nu}(k, k-q) S(k-q)\right] .
$$

In Eqs.(1) and (3) $\Gamma^{\nu}(k, p)$ is the dressed fermion-photon vertex which should satisfy the Ward-Takahashi identity

$$
(k-p)_{\mu} \Gamma^{\mu}(k, p)=S^{-1}(k)-S^{-1}(p)
$$

Eqs.(1) and (3) for the full fermion and photon propagators should be solved together with the equation for the full vertex function $\Gamma^{\mu}(k, p)$ 


$$
\begin{aligned}
\Gamma_{a b}^{\mu}(k, p)=\gamma_{a b}^{\mu}+ & \frac{i e^{2}}{(2 \pi)^{3}} \int_{M} \mathrm{~d}^{3} q\left[S(k+q) \Gamma^{\mu}(k+q, p+q) S(p+q)\right]_{d c} \\
& \times K_{c d, b a}(p+q, k+q, q),
\end{aligned}
$$

which in its turn contains an unknown kernel $K(p, k, q)$ (fermion-antifermion scattering amplitude), and so on. Because the system of SDE is in fact an infinite chain of integral equations for $n$-point Green's functions, we are forced to use some truncation scheme, which we require to be consistent with WT identity. One can verify that if we take the bare vertex approximation $\left(\Gamma^{\mu}=\gamma^{\mu}\right)$ in Eq.(1) and use the ladder approximation for the kernel $K(p, k, q)$ :

$$
K(p+q, k+q, q)_{c d, b a}=\gamma_{a d}^{\lambda} \gamma_{c b}^{\rho} D_{\lambda \rho}(q),
$$

we get a closed system of SDE consistent with WT identity (4) (Eqs.(2),(3) remain unaltered), see Fig. 1. Indeed, let us multiply Eq.(5) with the kernel (6) by $(k-p)_{\mu}$, then taking into account Eq.(1) (with $\Gamma^{\nu}=\gamma^{\nu}$ ) we write

$$
\begin{aligned}
& \hat{k}-\hat{p}=S^{-1}(k)-S^{-1}(p) \\
& +\frac{i e^{2}}{(2 \pi)^{3}} \int_{M} \mathrm{~d}^{3} q \gamma^{\mu} S(q+k) \gamma^{\nu} D_{\mu \nu}(q)-\frac{i e^{2}}{(2 \pi)^{3}} \int_{M} \mathrm{~d}^{3} q \gamma^{\mu} S(q+p) \gamma^{\nu} D_{\mu \nu}(q),
\end{aligned}
$$

which allows us to reduce Eq.(5) to the following:

$$
\begin{aligned}
& (k-p)_{\mu} \Gamma^{\mu}(k, p)-S^{-1}(k)+S^{-1}(p)=\frac{i e^{2}}{(2 \pi)^{2}} \int_{M} \mathrm{~d}^{3} q \gamma^{\lambda} S(k+q) \\
& \times\left[(k-p)_{\mu} \Gamma^{\mu}(k+q, p+q)-S^{-1}(k+q)+S^{-1}(p+q)\right] \\
& \times S(p+q) \gamma^{\rho} D_{\lambda \rho}(q) .
\end{aligned}
$$

It is evident now that Eq.(8) has a solution satisfying WT identity Eq.(4).

Due to the Ward-Takahashi identity, the vacuum polarization tensor Eq.(3) has the form

$$
\Pi_{\mu \nu}(q)=\left(-g_{\mu \nu} q^{2}+q_{\mu} q_{\nu}\right) \Pi\left(q^{2}\right)
$$

where $\Pi\left(q^{2}\right)$ is the vacuum polarization (we recall that we use four-component spinors).

The general form for the full fermion propagator can be expressed as

$$
S(p)=\frac{Z\left(p^{2}\right)}{\hat{p}-M\left(p^{2}\right)},
$$

where $Z$ is the fermion wave function and $M$ is the mass function. Then the equation for the mass function is

$$
\frac{M\left(p^{2}\right)}{Z\left(p^{2}\right)}=\frac{i e^{2}}{(2 \pi)^{3}} \int_{M} \mathrm{~d}^{3} k \gamma^{\mu} \gamma^{\nu} D_{\mu \nu}(k-p) \frac{M\left(k^{2}\right) Z\left(k^{2}\right)}{k^{2}-M^{2}\left(k^{2}\right)},
$$

and for the fermion wave function 


$$
\hat{p}\left(1-\frac{1}{Z\left(p^{2}\right)}\right)=\frac{i e^{2}}{(2 \pi)^{3}} \int_{M} \mathrm{~d}^{3} k \gamma^{\mu} \hat{k} \gamma^{\nu} D_{\mu \nu}(k-p) \frac{Z\left(k^{2}\right)}{k^{2}-M^{2}\left(k^{2}\right)} .
$$

The general tensor structure of the vertex contains eight scalar functions [7]; thus, together with the functions $Z\left(p^{2}\right), M\left(p^{2}\right)$ and $\Pi\left(p^{2}\right)$, we can write down a coupled system of integral equations for eleven unknown scalar functions. Perhaps, this is the simplest truncated set of SDE consistent with the WT identity. We emphasize that such a truncation scheme can be used also in four-dimensional QED with one (though important) difference: due to the superficial linear divergence of the electron self-energy we should take a photon momentum as an integration variable in order to avoid shifting variables. Certainly, this scheme does not satisfy the requirement of multiplicative renormalizability, but that property is a feature of the whole theory rather than of any approximation scheme.

Now, it is well known that $Z$ is a gauge-dependent function, and we can use the freedom of choosing a convenient gauge to make the function $Z\left(p^{2}\right)=1$. To achieve this we use a nonlocal gauge fixing procedure $[13,14]$. The photon propagator in a general nonlocal covariant gauge is defined as [19]

$$
D_{\mu \nu}(q)=\left(-g_{\mu \nu}+\frac{q_{\mu} q_{\nu}}{q^{2}}\right) \frac{1}{q^{2}} \frac{1}{1+\Pi\left(q^{2}\right)}-\xi\left(q^{2}\right) \frac{q_{\mu} q_{\nu}}{q^{4}},
$$

with $\xi\left(q^{2}\right)$ being a function of momentum $q$ rather than a constant. Simmons proved [13] that a suitable form for $\xi\left(q^{2}\right)$ is the following:

$$
\xi\left(q^{2}\right)=\frac{2}{1+\Pi\left(q^{2}\right)}-\frac{2}{q^{2}} \int_{0}^{q^{2}} \frac{\mathrm{d} v}{1+\Pi(v)} .
$$

With this gauge function $\xi\left(q^{2}\right)$, the right-hand side of Eq.(12) vanishes when averaged over the direction of $\boldsymbol{k}$.

For Eq.(11) we obtain, in Euclidean formulation,

$$
M\left(p^{2}\right)=\frac{e^{2}}{(2 \pi)^{3}} \int_{E} \mathrm{~d}^{3} k \frac{M\left(k^{2}\right)}{k^{2}+M^{2}\left(k^{2}\right)} \frac{1}{q^{2}}\left[\frac{2}{1+\Pi\left(q^{2}\right)}+\xi\left(q^{2}\right)\right],
$$

where $q^{\mu}=k^{\mu}-p^{\mu}$.

The system of Eqs.(2), (3), (5), (6) and (15) is still far too complicated for an analytical study, and we postpone the full investigation of it to the future. Here we note only that the mass function $M\left(p^{2}\right)$ is connected to scalar functions from the vertex through the vacuum polarization $\Pi\left(q^{2}\right)$. If we assume that this connection is not crucial, we can take the oneloop approximation for the vacuum polarization, replacing the vertex $\Gamma^{\nu}$ by the bare vertex $\gamma^{\nu}$, and the running mass function $M\left(p^{2}\right)$ by its value $M(0)$. This approximation, though keeping the transversality condition for $\Pi_{\mu \nu}(q)$, leads to violation of the WT identity Eq.(4), however, in the nonlocal gauge the WT identity is still approximately satisfied.

Thus the vacuum polarization in the one-loop approximation takes the form (with Euclidean momentum $q$ ):

$$
\Pi\left(q^{2}\right)=\frac{N_{f} e^{2}}{4 \pi q^{2}}\left[2 m+\frac{q^{2}-4 m^{2}}{q} \arctan \left(\frac{q}{2 m}\right)\right],
$$


where in our case we take $m \equiv M\left(p^{2}=0\right)$. Taking massless fermion loops in vacuum polarization, we find

$$
\Pi\left(q^{2}\right)=\frac{\alpha}{q}, \quad \alpha=\frac{N_{f} e^{2}}{8}
$$

\section{COORDINATE SPACE FORMULATION}

First we study Eq.(15) in the linearized form, i.e.

$$
M\left(p^{2}\right)=\frac{e^{2}}{(2 \pi)^{3}} \int_{E} \mathrm{~d}^{3} k \frac{M\left(k^{2}\right)}{k^{2}+m^{2}} \frac{1}{q^{2}}\left[\frac{2}{1+\Pi\left(q^{2}\right)}+\xi\left(q^{2}\right)\right],
$$

where we have replaced $M\left(k^{2}\right)$ in the denominator by $m \equiv M(0)$. In terms of the coordinate space function

$$
\psi(r) \equiv \int \frac{\mathrm{d}^{3} p}{(2 \pi)^{3}} \frac{M\left(p^{2}\right)}{p^{2}+m^{2}} \mathrm{e}^{i p \cdot r}
$$

the equivalent of Eq.(18) is

$$
\left(-\nabla^{2}+m^{2}\right) \psi(r)=e^{2} \int \frac{\mathrm{d}^{3} p \mathrm{~d}^{3} k}{(2 \pi)^{6}} \frac{M\left(k^{2}\right)}{k^{2}+m^{2}} \frac{1}{q^{2}}\left[\frac{2}{1+\Pi\left(q^{2}\right)}+\xi\left(q^{2}\right)\right] \mathrm{e}^{i p \cdot r} .
$$

Performing a shift of integration variable, $p \rightarrow p+k$, we find a Schrödinger-like equation for the function $\psi(r)$ :

$$
\left(-\nabla^{2}+V(r)\right) \psi(r)=-m^{2} \psi(r)
$$

where the potential $V(r)$ is defined as

$$
V(r)=-\frac{e^{2}}{2 \pi^{2} r} \int_{0}^{\infty} \mathrm{d} p \frac{\sin p r}{p}\left[\frac{2}{1+\Pi\left(p^{2}\right)}+\xi\left(p^{2}\right)\right] .
$$

In order to simplify (22), for both the massless and massive case we approximate the vacuum polarization by

$$
\Pi\left(q^{2}\right)=\frac{\alpha}{q+\beta}
$$

where $\beta=\sigma m, \sigma$ being some fitting constant, and $\alpha=N_{f} e^{2} / 8$. The massless vacuum polarization corresponds to putting $\sigma=0$, while the value $\sigma=3 \pi / 4$ corresponds to approximating both infrared and ultraviolet behaviors of the exact one-loop expression for $\Pi\left(q^{2}\right)$. The above approximation allows us to perform the integral in $\xi\left(q^{2}\right)$ analytically, i.e. with (23) 


$$
\int_{0}^{q^{2}} \frac{\mathrm{d} v}{1+\Pi(v)}=q^{2}-2 \alpha q+2 \alpha(\alpha+\beta) \log \left(1+\frac{q}{\alpha+\beta}\right) .
$$

With $\alpha=1$, i.e. $e^{2}=8 / N_{f}$, the potential can be expressed as

$$
\begin{aligned}
V(r)=- & \frac{8}{\pi^{2} N_{f} r} \int_{0}^{\infty} \mathrm{d} x \frac{\sin x r}{x} \\
& \times\left[\frac{2(x+\beta)}{x+1+\beta}+\frac{2}{x}-1-\frac{2(1+\beta)}{x^{2}} \log \left(1+\frac{x}{1+\beta}\right)\right] .
\end{aligned}
$$

The last expression can be rewritten in a more convenient form:

$$
\begin{aligned}
& V(r)=-\frac{4}{\pi N_{f} r} \frac{\beta}{\beta+1}-\frac{16}{\pi^{2} N_{f} r} \int_{0}^{\infty} \mathrm{d} x \sin x r \\
& \times\left[\frac{1}{(1+\beta)(x+1+\beta)}+\frac{1}{x^{2}}-\frac{1}{2(1+\beta) x}-\frac{(1+\beta)}{x^{3}} \log \left(1+\frac{x}{1+\beta}\right)\right] .
\end{aligned}
$$

We can get rid of the oscillatory behavior of the sine by performing a contour rotation in the lower right quadrant of the complex-plane of $x$. So

$$
\begin{aligned}
V(r)= & -\frac{4}{\pi N_{f} r} \frac{\beta}{1+\beta}-\frac{16}{\pi^{2} N_{f} r} \int_{0}^{\infty} \mathrm{d} y \exp (-y r) \\
& \times\left[\frac{1}{y^{2}+(1+\beta)^{2}}-\frac{1}{y^{2}}+\frac{(1+\beta)}{y^{3}} \arctan \frac{y}{1+\beta}\right] .
\end{aligned}
$$

Let us consider first the case of vacuum polarization by massless fermions, i.e. $m=0$, which coincides precisely with the approximation used by Appelquist, Nash and Wijewardhana [2] in momentum space. The potential (27) takes the form

$$
V(r)=-\frac{16}{\pi^{2} N_{f} r} \int_{0}^{\infty} d y \exp (-y r)\left[\frac{1}{y^{2}+1}-\frac{1}{y^{2}}+\frac{1}{y^{3}} \arctan y\right] .
$$

This potential behaves at large distances as

$$
V(r) \sim-\frac{\lambda_{1}}{r^{2}}, \quad \lambda_{1}=\frac{32}{3 \pi^{2} N_{f}},
$$

whereas at small distances it has Coulomb-like behavior:

$$
V(r) \sim-\frac{\lambda_{2}}{r}, \quad \lambda_{2}=\frac{4}{\pi N_{f}} .
$$

From the theory of the Schrödinger equation for such kinds of potential, it is known $[20,21]$ that there is an infinite number of bound states if $\lambda_{1}>1 / 4\left(N_{f}<N_{c}=128 / 3 \pi^{2}\right)$. However a finite number of bound states might exist for $\lambda_{1}<1 / 4$ as well. Let us show that for the 
potential under consideration there are no bound states at all for $\lambda_{1}<1 / 4$. Indeed, one can prove the following inequality

$$
V(r)>-\frac{\lambda_{1}}{r} \int_{0}^{\infty} \mathrm{d} y \frac{\exp (-y r)}{y^{2}+1}>-\frac{\lambda_{1}}{r^{2}} .
$$

Since the Schrödinger equation

$$
\left(-\nabla^{2}-\frac{\lambda_{1}}{r^{2}}\right) \psi(r)=-m^{2} \psi(r)
$$

has no bound states for $\lambda_{1}<\frac{1}{4}$ [20], it is evident from the inequality (31) that the Schrödinger equation (21) with the potential $V(r)(28)$ has none either. Thus we have proved that the critical number of fermions,

$$
N_{c}=\frac{128}{3 \pi^{2}} \approx 4.32304
$$

does indeed correspond to the appearance of an infinite number of bound states in an effective Schrödinger equation in $r$-space, if we consider the vacuum polarization by massless fermions. The critical number of fermions (33) coincides with the result obtained by Nash in a different way [22] and that was claimed as being gauge invariant to leading order in the $1 / N_{f}$ expansion.

The quasi-classical approach gives the following spectrum of bound states for potentials behaving at large distances as $-\lambda / r^{2}[21]$ :

$$
E_{n}=-m_{n}^{2} \sim-\exp \left[-\frac{2 \pi n}{\sqrt{\lambda_{1}-1 / 4}}\right]
$$

at $n>>1$, coinciding in fact with the result of Refs. [2,22]. As was pointed out in [2], this spectrum is closely connected with approximate scale invariance of $\mathrm{QED}_{3}$ at intermediate distances $1<r<1 / m^{1}$. However the quasi-classical theory does not tell us anything about lower levels of the Schrödinger equation. It is clear, also, that the proof given above breaks down in the case of massive fermion vacuum polarization, since the potential (27) has a Coulomb-like tail at large distances. The latter is simply a reflection of the fact that including a dressed fermion propagator restores the confinement property of $\mathrm{QED}_{3}$ in the sense that a logarithmic term reappears in the real potential at large $r$ [11]. In the next section we shall solve the Schrödinger equation with the potential (27) and study the scaling properties of the dynamical mass near $N_{c}$.

\footnotetext{
${ }^{1}$ We note that in $\mathrm{QED}_{4}$ it is exactly the scale invariance of the ladder and/or quenched approximation which is responsible for exponential-like behavior of a dynamical fermion mass. For physical explanation of this fact see Ref. [23]. Further discussion of the role of scale invariance in $\mathrm{QED}_{4}$ can be found in Refs. [24-26].
} 


\section{SCALING PROPERTIES NEAR CRITICALITY}

In this section we study the scaling properties of the dynamical mass near $N_{c}$, for both massless and massive vacuum polarization. For large $r, V(r)(27)$ has the following form

$$
V(r) \approx-\frac{\lambda_{1}}{r^{2}}-\frac{\lambda_{3} m}{r}, \quad r \gg \mathcal{O}(1)
$$

where

$$
\lambda_{3}=\frac{4 \sigma}{\pi N_{f}}, \quad \beta=\sigma m .
$$

In deriving this we neglected terms $\beta$ with respect to 1 , since we consider $V(r)$ near $N_{c}$, where $m$ is very small (and so is $\beta$ ). For large $r$ we have thus a $-1 / r^{2}$ potential with a small Coulomb potential which starts to contribute for $r \geq 1 / \beta$. For small $r, V(r)$ behaves like a Coulomb potential plus a constant part

$$
V(r) \approx-\frac{\lambda_{2}}{r}+\frac{3}{2} \lambda_{1}, \quad r \ll \mathcal{O}(1)
$$

We split our problem into two regions, for large $r$, and small $r$. Neglecting angular dependence, which is not relevant for our purpose, we obtain

$$
\begin{aligned}
\frac{\mathrm{d}^{2} \psi}{\mathrm{d} r^{2}}+\frac{2}{r} \frac{\mathrm{d} \psi}{\mathrm{d} r}+\left(\frac{\lambda_{1}}{r^{2}}+\frac{\lambda_{3} m}{r}-m^{2}\right) \psi & =0, & & r \gg 1, \\
\frac{\mathrm{d}^{2} \psi}{\mathrm{d} r^{2}}+\frac{2}{r} \frac{\mathrm{d} \psi}{\mathrm{d} r}+\left(\frac{\lambda_{2}}{r}-\tilde{m}^{2}\right) \psi & =0, & & r \ll 1,
\end{aligned}
$$

where $\tilde{m}^{2}=\frac{3}{2} \lambda_{1}+m^{2}$. With $\psi(r)=f(r) / r$, the equations can be expressed as Whittaker equations

$$
\begin{aligned}
& \frac{\mathrm{d}^{2} f}{\mathrm{~d} x^{2}}+\left(\frac{\lambda_{1}}{x^{2}}+\frac{\lambda_{3}}{2 x}-\frac{1}{4}\right) f=0, \quad x=2 m r, \quad r \gg 1, \\
& \frac{\mathrm{d}^{2} f}{\mathrm{~d} x^{2}}+\left(\frac{\lambda_{2}}{2 \tilde{m} x}-\frac{1}{4}\right) f=0, \quad x=2 \tilde{m} r, \quad r \ll 1,
\end{aligned}
$$

where the general form of a Whittaker differential equation is defined as [27]

$$
\frac{\mathrm{d}^{2} f}{\mathrm{~d} x^{2}}+\left(\frac{\frac{1}{4}-\mu^{2}}{x^{2}}+\frac{\kappa}{x}-\frac{1}{4}\right) f=0 .
$$

Two independent solutions of (42) are the Whittaker functions

$$
\begin{aligned}
& M_{\kappa, \mu}(x)=\mathrm{e}^{-\frac{x}{2}} x^{\frac{c}{2}} \Phi(a, c ; x), \\
& W_{\kappa, \mu}(x)=\mathrm{e}^{-\frac{x}{2}} x^{\frac{c}{2}} \Psi(a, c ; x),
\end{aligned}
$$

where 


$$
\begin{aligned}
& \kappa=\frac{c}{2}-a, \\
& \mu=\frac{c}{2}-\frac{1}{2},
\end{aligned}
$$

$\Psi$ and $\Phi$ are confluent hypergeometric functions. The solution of Eq.(38) that is regular at $r=\infty$, has the form

$$
\psi_{1}(r)=\mathrm{e}^{-m r} r^{-\frac{1}{2}+i \nu} \Psi\left(\frac{1}{2}-\frac{\lambda_{3}}{2}+i \nu, 1+2 i \nu ; 2 m r\right), \quad r \gg 1,
$$

where $\nu=\sqrt{\lambda_{1}-\frac{1}{4}}$, which vanishes when $N_{f} \rightarrow N_{c}$. Eq.(39) has a solution of the type (43), which is regular at $r=0$ :

$$
\psi_{2}(r)=\mathrm{e}^{-\tilde{m} r} \Phi\left(1-\frac{\lambda_{2}}{2 \tilde{m}}, 2 ; 2 \tilde{m} r\right), \quad r \ll 1 .
$$

Since these two different solutions represent one solution of our original problem (21), we have to match them at some intermediate point $r=r_{0}=\mathcal{O}(1)$. A choice of $r_{0}$ corresponding to Appelquist et al. would be $r_{0}=1 / \alpha=1$. But for the time being we keep an arbitrary matching point. As matching condition we take

$$
\frac{\mathrm{d}}{\mathrm{d} r}\left[\log \frac{\psi_{2}(r)}{\psi_{1}(r)}\right]_{r=r_{0}}=0
$$

With

$$
\begin{aligned}
\Phi^{\prime}(a, c ; x) & =\frac{a}{c} \Phi(a+1, c+1 ; x), \\
\Psi^{\prime}(a, c ; x) & =-a \Psi(a+1, c+1 ; x),
\end{aligned}
$$

the matching condition (49) reads

$$
\begin{aligned}
& 2 m\left(\frac{1}{2}-\frac{\lambda_{3}}{2}+i \nu\right) \frac{\Psi\left(\frac{3}{2}-\frac{\lambda_{3}}{2}+i \nu, 2+2 i \nu ; 2 m r_{0}\right)}{\Psi\left(\frac{1}{2}-\frac{\lambda_{3}}{2}+i \nu, 1+2 i \nu ; 2 m r_{0}\right)}= \\
& =-\frac{1}{r_{0}}\left(\frac{1}{2}-i \nu\right)-m+\tilde{m}-\left(\tilde{m}-\frac{\lambda_{2}}{2}\right) \frac{\Phi\left(2-\frac{\lambda_{2}}{2 \tilde{m}}, 3 ; 2 \tilde{m} r_{0}\right)}{\Phi\left(1-\frac{\lambda_{2}}{2 \tilde{m}}, 2 ; 2 \tilde{m} r_{0}\right)} .
\end{aligned}
$$

Since we consider small $m$, the dependence on $m$ on right-hand side of the previous equation vanishes. We define

$$
d=\tilde{m}-\left(\tilde{m}-\frac{\lambda_{2}}{2}\right) \frac{\Phi\left(2-\frac{\lambda_{2}}{2 \tilde{m}}, 3 ; 2 \tilde{m} r_{0}\right)}{\Phi\left(1-\frac{\lambda_{2}}{2 \tilde{m}}, 2 ; 2 \tilde{m} r_{0}\right)}
$$

with $\tilde{m}=\sqrt{\frac{3 \lambda_{1}}{2}}$. Now $2 m r_{0} \ll 1$, so for the $\Psi$-function we can use the asymptotic form for small argument, i.e. 


$$
\Psi(a, c ; x) \underset{x \rightarrow 0}{\approx} \frac{\Gamma(1-c)}{\Gamma(a-c+1)}+\frac{\Gamma(c-1)}{\Gamma(a)} x^{1-c} .
$$

The matching condition then reads

$$
\begin{aligned}
& \left(\frac{1}{2}-\frac{\lambda_{3}}{2}+i \nu\right)\left[\frac{\Gamma(-1-2 i \nu)}{\Gamma\left(\frac{1}{2}-\frac{\lambda_{3}}{2}-i \nu\right)}\left(2 m r_{0}\right)^{1+i \nu}+\frac{\Gamma(1+2 i \nu)}{\Gamma\left(\frac{3}{2}-\frac{\lambda_{3}}{2}+i \nu\right)}\left(2 m r_{0}\right)^{-i \nu}\right]= \\
= & \left(d r_{0}-\frac{1}{2}+i \nu\right)\left[\frac{\Gamma(-2 i \nu)}{\Gamma\left(\frac{1}{2}-\frac{\lambda_{3}}{2}-i \nu\right)}\left(2 m r_{0}\right)^{i \nu}+\frac{\Gamma(2 i \nu)}{\Gamma\left(\frac{1}{2}-\frac{\lambda_{3}}{2}+i \nu\right)}\left(2 m r_{0}\right)^{-i \nu}\right],
\end{aligned}
$$

which can be rewritten as

$$
\begin{aligned}
& \left(\frac{1}{2}+i \nu\right) \frac{\Gamma(2 i \nu)}{\Gamma\left(\frac{1}{2}-\frac{\lambda_{3}}{2}+i \nu\right)}\left(2 m r_{0}\right)^{-i \nu}+\left(\frac{1}{2}-i \nu\right) \frac{\Gamma(-2 i \nu)}{\Gamma\left(\frac{1}{2}-\frac{\lambda_{3}}{2}-i \nu\right)}\left(2 m r_{0}\right)^{i \nu}= \\
= & d r_{0}\left[\frac{\Gamma(2 i \nu)}{\Gamma\left(\frac{1}{2}-\frac{\lambda_{3}}{2}+i \nu\right)}\left(2 m r_{0}\right)^{-i \nu}+\frac{\Gamma(-2 i \nu)}{\Gamma\left(\frac{1}{2}-\frac{\lambda_{3}}{2}-i \nu\right)}\left(2 m r_{0}\right)^{i \nu}\right] .
\end{aligned}
$$

With the use of

$$
a \mathrm{e}^{i \phi}+\bar{a} \mathrm{e}^{-i \phi}=2|a| \cos (\phi+\theta), \quad \theta=\arg (a),
$$

the equation (56) can be reduced to the following one

$$
\tan \left(\nu \log \frac{1}{2 m r_{0}}+\Sigma(\nu)\right)=\frac{2 \nu}{2 d r_{0}-1}
$$

where

$$
\Sigma(\nu)=\arg \left(\frac{\Gamma(1+2 i \nu)}{\Gamma\left(\frac{1}{2}-\frac{\lambda_{3}}{2}+i \nu\right)}\right) .
$$

Eq.(58) then gives for $m$ :

$$
m=\exp \left[-\frac{n \pi}{\nu}-\log \left(2 r_{0}\right)+\frac{1}{\nu} \arctan \frac{2 \nu}{1-2 d r_{0}}+\frac{1}{\nu} \Sigma(\nu)\right],
$$

with $n$ a positive integer. In the limit $\nu \rightarrow 0$, Eq.(60) takes the form

$$
m=\exp \left(-\frac{2 \pi n}{\sqrt{\frac{N_{c}}{N_{f}}-1}}+b\right)
$$

where

$$
b=-\log \left(2 r_{0}\right)+\frac{2}{1-2 d r_{0}}-2 \gamma+\psi\left(\frac{1}{2}-\frac{\lambda_{3}}{2}\right),
$$

with $\psi$ the di-gamma function, $\psi(z)=\Gamma^{\prime}(z) / \Gamma(z)$, and where $\gamma$ is the Euler constant. Only the solution with the largest value of $m<1(n=1)$ corresponds to the ground state, since it has the lowest energy. 
With the help of Mathematica we can calculate $b$ explicitly for various values of the parameter $\sigma\left(r_{0}=1\right)$. With $\nu=0$

$$
\lambda_{1}=\frac{1}{4}, \quad \lambda_{2}=\frac{3 \pi}{32}, \quad \lambda_{3}=\frac{3 \pi \sigma}{32}, \quad \tilde{m}=\sqrt{\frac{3}{8}}
$$

this gives

$$
\begin{aligned}
& \text { massless fermion loops: } \sigma=0, \quad b=2.214 \text {, } \\
& \text { massive fermion loops: } \quad \sigma=\frac{3 \pi}{4}, \quad b=7.136 \text {. }
\end{aligned}
$$

Thus we have found that for a vacuum polarization with massive fermions, the infrared dynamical mass $m$ obeys an exponential scaling law near the critical point $N_{c}$ despite the presence of a Coulomb tail in the potential (27). This means that chiral symmetry breaking takes place essentially at intermediate distances $1<r<1 / m$. For the massless vacuum

polarization, this was first pointed out by Appelquist et al. [2]. The main difference between a vacuum polarization with massive (parameter $\sigma \neq 0$ ) or with massless fermions $(\sigma=0)$ lies in the scaling factor $b$, which is larger in the case of massive fermion loops in the vacuum polarization. We note here that our real space approach automatically takes into account the ultra violet tail of the integral equation in momentum space to which an attention was paid in Ref. [28]. Moreover, in comparison with their massless vacuum polarization calculations, our analytical solution gives the scaling factor $b$ closer to exact computation of that from whole nonlinear equation (compare our $b=2.214$ with Kondo and Maris' $b=3.94$ [28] against exact $b=1.842$ ).

\section{NUMERICAL CALCULATIONS IN MOMENTUM SPACE}

In this section we discuss the numerical calculation of the scaling law of the dynamical mass near criticality for a vacuum polarization with respectively massive and massless fermion loops. The nonlinear DSE can be written in the following way, with $\alpha=1$

$$
M\left(p^{2}\right)=\frac{2}{\pi^{2} N_{f}} \int_{0}^{\infty} \mathrm{d} k \frac{k^{2} M\left(k^{2}\right)}{k^{2}+M^{2}\left(k^{2}\right)} K\left(p^{2}, k^{2}, m\right),
$$

where the kernel $K$ is given by

$$
K\left(p^{2}, k^{2}, m\right)=\int_{0}^{\pi} \mathrm{d} \theta \sin \theta \frac{4}{q^{2}}\left[\frac{1}{1+\Pi\left(q^{2}\right)}-\frac{1}{2} \int_{0}^{1} \frac{\mathrm{d} y}{1+\Pi\left(q^{2} y\right)}\right] .
$$

with $q^{2}=k^{2}+p^{2}-2 p k \cos \theta$, and we recall the expression for the one-loop vacuum polarization,

$$
\Pi\left(q^{2}\right)=\frac{2}{\pi q^{2}}\left[2 m+\frac{q^{2}-4 m^{2}}{q} \arctan \left(\frac{q}{2 m}\right)\right] .
$$


In the case of massless fermions in the vacuum polarization, the $y$-integration in (66) can be performed explicitly. It reduces to

$$
K\left(p^{2}, k^{2}, 0\right)=\int_{0}^{\pi} \mathrm{d} \theta \sin \theta \frac{4}{q^{2}}\left[\frac{q}{1+q}-\frac{1}{2}+\frac{1}{q}-\frac{1}{q^{2}} \log (1+q)\right],
$$

which does not depend on the dynamical mass, so that it only has to be calculated once. It is convenient to perform the calculations on a $\log p^{2}$ scale in order to see more details of the structure.

$$
M(x)=\frac{1}{\pi^{2} N_{f}} \int_{\log \mu^{2}}^{\log \Lambda^{2}} \mathrm{~d} t \frac{y \sqrt{y} M(y)}{y+M^{2}(y)} K(x, y, m)
$$

where $x=p^{2}$, and $y=k^{2}=\exp (t)$. We have introduced infrared and ultraviolet cutoffs, $\mu$ and $\Lambda$, respectively, which should satisfy $\mu \ll M(0)$, and $\Lambda \gg \alpha=1$.

The basic idea of our numerical calculations is that we solve Eq.(65) iteratively as an integral equation. First we calculate the kernel $K$ for all grid points $x$ and $y$ with some initial value for $m$. Then we iterate the integral equation until it has reached some convergence criterion. Then we recalculate the kernel with the new $m=M(0)$, and iterate again. This procedure we repeat to the point were the complete system has converged. To obtain a satisfactory numerical procedure, the integrals in $K$ are performed using Gauss-Legendre quadrature, which works well since our integrands in (69) are sufficiently smooth. For $M$ at the grid points $x$ we use cubic spline interpolation on a $\log x$-scale. The $t$-integration is performed using Gauss-Legendre quadrature between the grid points $x$,

$$
M\left(x_{i}\right)=\frac{1}{\pi^{2} N_{f}} \sum_{j=1}^{n-1} \int_{\log x_{j}}^{\log x_{j+1}} \mathrm{~d} t\left[\frac{y \sqrt{y} M(y)}{y+M^{2}(y)} K\left(x_{i}, y, m\right)\right], \quad i=1, \ldots, n,
$$

where $x_{1}=\mu^{2}$, and $x_{n}=\Lambda^{2}$. With this procedure we find that for calculation near the critical point $N_{c}$, we need at least 128 grid points for $x$. We have calculated the mass function $M(x)$ for a series of values of $N_{f}$ near $N_{c}$. For all cases it appears that the infrared $m$ obeys the exponential scaling law. This has been verified using a least squares fit for the numerical data. We have fitted the infrared mass $m$, as a function of $N_{f}$, with the following function

$$
\log m\left(N_{f}\right)=-\frac{2 \pi a}{\left(c / N_{f}-1\right)^{d}}+b
$$

where $a, b, c$, and $d$ are the parameters to be fitted. For the nonlinear massless case the least squares fitting of the data gives

$$
a=0.9907, \quad b=1.720, \quad c=4.32312, \quad d=0.5018,
$$

and with massive fermion loops

$$
a=1.055, \quad b=3.494, \quad c=4.32315, \quad d=0.4893,
$$


which is shown in Fig. 2. This fitting proves the exponential scaling law (61). To obtain values of $b$ and compare them with the analytic calculation we take $a=1, c=N_{c}=128 / 3 \pi^{2}$, $d=1 / 2$. The numerical results are:

$$
\begin{aligned}
\text { massless fermion loops: } & b=1.842, \\
\text { massive fermion loops: } & b=2.955 .
\end{aligned}
$$

We also investigated the difference between a linearized approximation (18) and the nonlinear DSE (15). Qualitively the linearized approximation is equivalent to the more realistic nonlinear equation. Quantitively the scaling factor $b$ is a few percent smaller for the linearized model.

In Fig.3 we have plotted the dynamical fermion mass function $M\left(p^{2}\right)$, computed for various values of the fermion number $N_{f}$. Apart of $N_{c}$ it has behavior reminiscent to that in the quenched approximation [9,10]: the mass function is constant at momenta $p \ll \alpha$ and decreases as $\alpha^{3} / p^{2}$ at large momenta. The mass function changes its behavior at momentum $p \simeq \alpha$, a natural scale of the model. Close to critical number of fermions, $N_{f} \lesssim N_{c}$, we observe three different regions in behavior of $M\left(p^{2}\right): M\left(p^{2}\right) \simeq m$ at $p \leq m, M\left(p^{2}\right) \sim$ $m^{3 / 2} / \sqrt{p}$ at $m \leq p \leq \alpha$ and $M\left(p^{2}\right) \sim m^{3} / p^{2}$ at $p>\alpha$. The scale $m$ is exponentially small in comparison with the intrinsic scale $\alpha$, see Eq.(71). Fig.3 shows convincingly how a hierarchy of scales can occur in a theory under specific conditions (in our case when $N_{f}$ is close to $N_{c}$ ). This might be important for further understanding a hierarchy of scales in unified theories.

\section{CONCLUSION AND DISCUSSION}

In the present paper we investigated $\chi \mathrm{SB}$ in $(2+1)$-dimensional QED with $N_{f}$ number of four-component Dirac fermions. We have pointed out one possible scheme for truncating an infinite chain of SDE, which is consistent with WT identity and which seems to have been overlooked so far. Further simplification of that coupled system of SDE led to studying the SD equation for a fermion mass function where the full vacuum polarization with dynamically massive fermions has been taken into account. The latter proves to be important for keeping the confinement property of the model at large distances. The study of the linearized equation for a fermion mass function has been performed in real space,and that allowed us to avoid making drastic approximations in order to get analytical results. Our analytical solution fits nicely numerical calculations of the full nonlinear integral equation for the mass function.

Further investigation of aforementioned truncated system of SDE is now in progress.

\section{ACKNOWLEDGEMENTS}

We would like to express sincere thanks to David Atkinson for many stimulating discussions and for correcting the manuscript. We thank Y. Hoshino, who took part in investigations at early stages of this work, for collaboration. We are grateful also to Pieter Maris and Volodya Miransky for valuable remarks. V.P.G. is grateful to the members of the Insti-

tute for Theoretical Physics of the University of Groningen for their hospitality. He wishes 
to acknowledge the Stichting FOM (Fundamental Onderzoek der Materie), financially supported by the Nederlandse Organisatie voor Wetenschappelijk Onderzoek, for its support. The work of one of us (V.P.G.) was supported in part by Grant INTAS-93-2058 "East-West network in constrained dynamical systems". 


\section{REFERENCES}

[1] T.W. Appelquist, M. Bowick, D. Karabali and L.C.R. Wijewardhana, Phys. Rev. D 33, 3704 (1986).

[2] T.W. Appelquist, D. Nash and L.C.R. Wijewardhana, Phys. Rev. Lett. 60, 2575 (1988).

[3] E. Dagotto, J.B. Kogut, A. Kocić, Phys. Rev. Lett. 62, 1083 (1989).

[4] E. Dagotto, A. Kocić and J.B. Kogut, Nucl. Phys. B 334, 279 (1990).

[5] M.R. Pennington and S.P. Webb, Hierarchy of scales in three dimensional QED, BNL40886, 1988 (unpublished); M.R. Pennington and D. Walsh, Phys. Lett. B 253, 246 (1991).

[6] D. Atkinson, P.W. Johnson and M.R. Pennington, Dynamical mass generation in threedimensional QED, BNL-41615, 1988 (unpublished).

[7] D.C. Curtis and M.R. Pennington, Phys. Rev. D 42, 4165 (1990).

[8] C.D. Roberts and A.G. Williams, Progr. Part. and Nucl. Phys. 33, 477 (1994).

[9] Y. Hoshino and T. Matsuyama, Phys. Lett. B 222, 493 (1989).

[10] K.-I. Kondo and H. Nakatani, Mod. Phys. Lett. A 5, 407 (1990).

[11] C.J. Burden, J. Praschifka and C.D. Roberts, Phys. Rev. D 46, (1992).

[12] P. Maris, Confinement and Complex Singularities in $Q E D_{3}$, DPNU-95-20, 1995; hep$\mathrm{ph} / 9508323$.

[13] E.H. Simmons, Phys. Rev. D 42, 2933 (1990).

[14] T. Kugo and M.G. Mitchard, Phys. Lett. B 282, 162 (1992).

[15] D. Atkinson, P.W. Johnson and K. Stam, Phys. Lett. B201, 105 (1988).

[16] K.-I. Kondo, Intern. J. Mod. Phys. A 7, 7239 (1992).

[17] V.A. Miransky, V.P. Gusynin and Yu.A. Sitenko, Phys. Lett. B 100, 157 (1981).

[18] T. Goldman and R.W. Haymayker, Phys. Lett. B 100, 276 (1981).

[19] L.D. Landau and E.M. Lifshitz, Relativistic Quantum Theory, II, Pergamon Press, Oxford, 1974.

[20] L.D. Landau and E.M. Lifshitz, Quantum Mechanics, Pergamon Press, Oxford, 1977.

[21] A.M. Perelomov and V.S. Popov, Theor. and Math. Physics, 4, 664 (1970).

[22] D. Nash, Phys. Rev. Lett. 62, 3024 (1989).

[23] P.I. Fomin, V.P. Gusynin, V.A. Miransky and Yu.A. Sitenko, Rivista Nuovo Cim. 6, 1 (1983).

[24] W.A. Bardeen, C. Leung and S. Love, Nucl. Phys. B 273, 649 (1989).

[25] B. Holdom, Phys. Rev. Lett. 62, 997 (1989).

[26] D. Atkinson, V.P. Gusynin and P. Maris, Phys. Lett. B 303, 157 (1993).

[27] A. Erdélyi et al., Higher Transcendental Functions, vol. I., McGraw-Hill, New York, 1953.

[28] K.-I. Kondo and P. Maris, Phys.Rev. D 52, 1212 (1995). 


\section{FIGURES}

FIG. 1. Truncated system of Schwinger-Dyson equations.

FIG. 2. The numerical results for $M(0)$ as function of $N_{f}$ for massive and massless fermion loops in vacuum polarization.

FIG. 3. Dynamical mass function $M\left(p^{2}\right)$ for $N_{f}=1.0,2.0,3.0$, 4.0. 
Figure 1
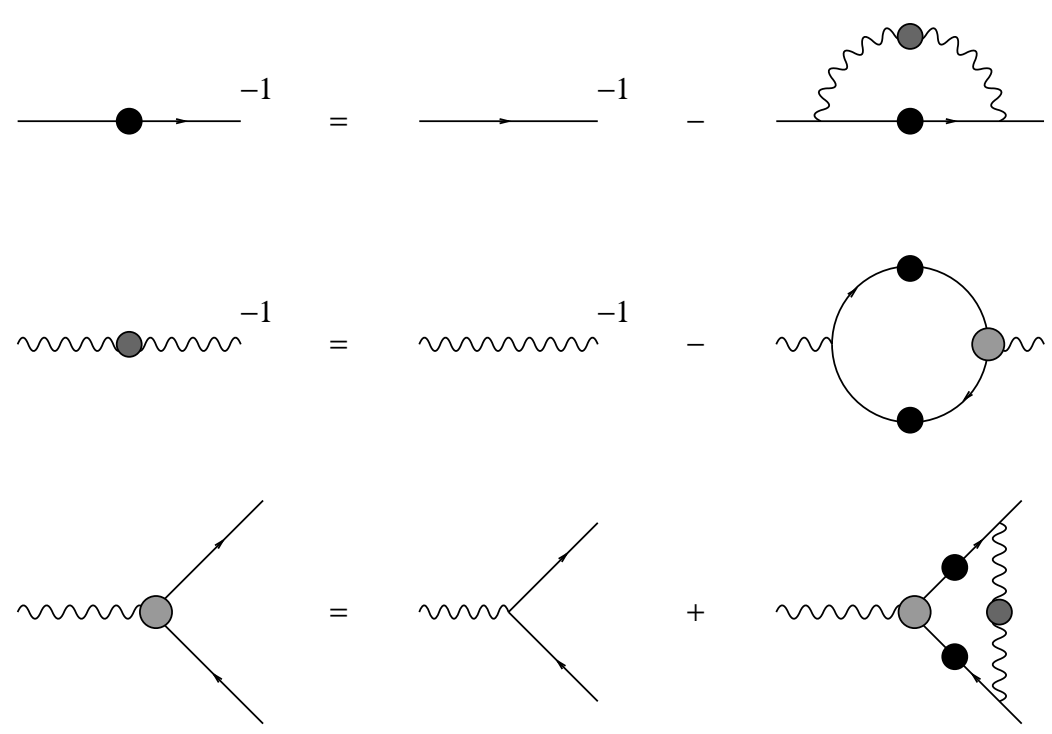
Figure 2

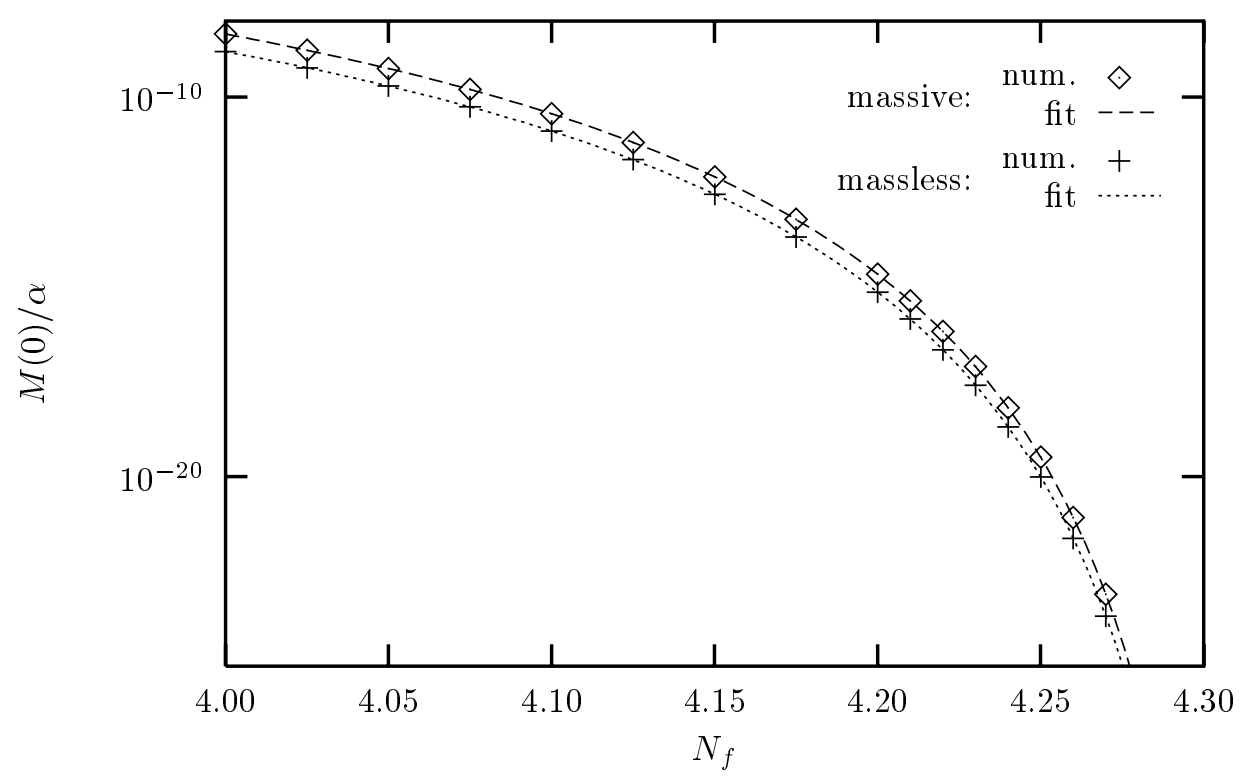


Figure 3

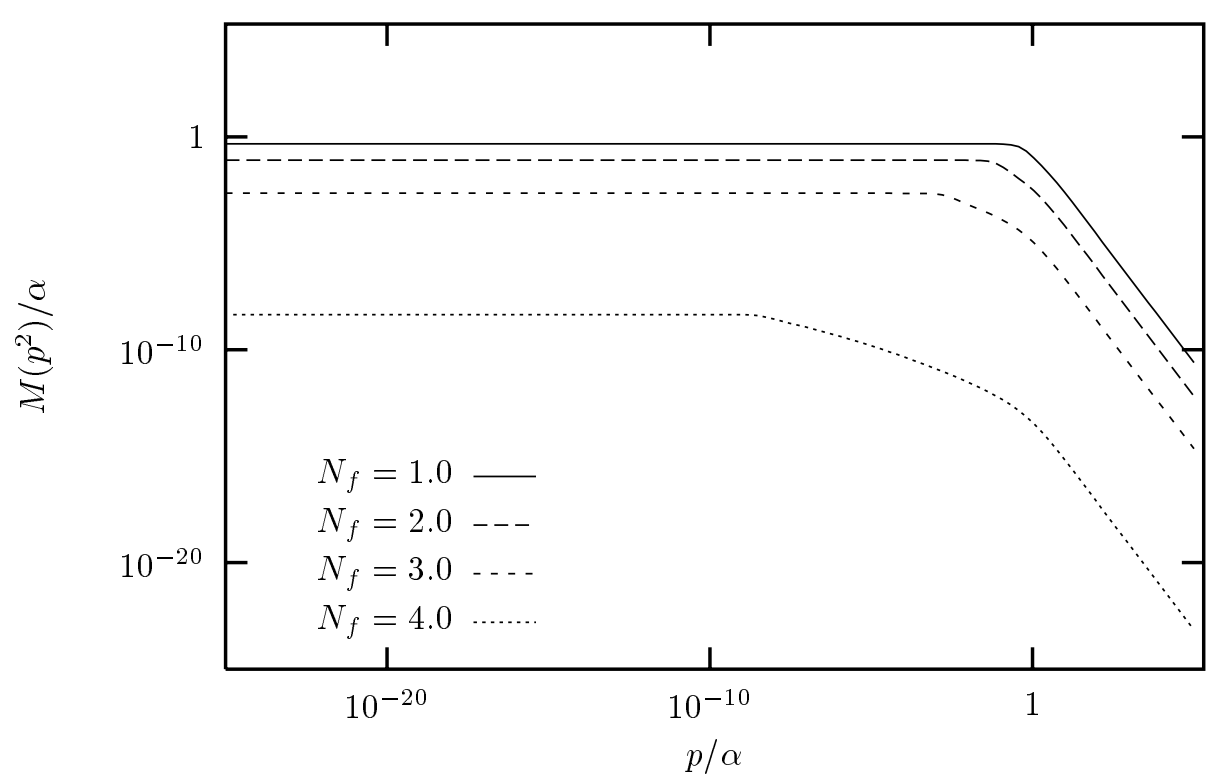

